\title{
Risk factors for side-effects of isoniazid, rifampin and pyrazinamide in patients hospitalized for pulmonary tuberculosis
}

\author{
T. Schaberg, K. Rebhan, H. Lode
}

Risk factors for side-effects of isoniazid, rifampin and pyrazinamide in patients hospitalized for pulmonary tuberculosis. T. Schaberg, K. Rebhan, H. Lode. @ERS Journals Ltd 1996.

ABSTRACT: The aim of this study was to determine the current incidence of sideeffects severe enough to cause intolerance of standard antituberculosis therapy with isoniazid, rifampin and pyrazinamide in patients hospitalized as a result of pulmonary tuberculosis.

Five hundred and nineteen patients with proven pulmonary tuberculosis, who initially received standard antituberculosis therapy, were retrospectively studied in the department of infectious diseases in a teaching chest hospital. The incidence of severe side-effects related to the therapy, which led to the definitive termination of one of the three standard drugs, was measured and the risk factors for intolerance were analysed.

Final termination of either isoniazid, rifampin or pyrazinamide because of severe side-effects was necessary in 121 of the 519 patients $(23 \%)$. The most severe sideeffects leading to final termination of one drug were hepatotoxicity $(11 \%)$, exanthema $(6 \%)$, and arthralgia (2\%). Pyrazinamide showed more severe side-effects $(15 \%)$ than isoniazid $(7 \%)$ and rifampin $(1.5 \%)$. Significant risk factors for intolerance of the standard therapy following a multivariate analysis were a history of hepatitis (odds ratio $(O R) 3.4 ; 95 \%$ confidence interval $(95 \% \mathrm{CI}) 1.6-7.6 ; p=0.0026)$ and an age $\geq 60$ yrs (OR 1.9; 95\% CI 1.2-3.2; $p=0.017)$. Both of these risk factors were also significantly associated with the intolerance of pyrazinamide (history of hepatitis: OR 2.5; 95\% CI 1.4-4.3; $p=0.0045$; age $\geq 60$ yrs: OR 2.1, 95\% CI 1.3-3.5; $\mathrm{p}=0.0029$ ) but not of isoniazid and rifampin.

The side-effects of standard antituberculosis therapy are frequent in hospitalized patients aged $\geq 60$ yrs or with a history of previous hepatitis, and are probably due to pyrazinamide rather than to isoniazid or rifampin.

Eur Respir J., 1996, 9, 2026-2030.
Section of Infectious Diseases and Immunology, Chest Hospital Heckeshorn (affiliated with Freie Universität Berlin Berlin), Germany.

Correspondence: T. Schaberg

Pneumology I

Section of Infectious Diseases and

Immunology

Chest Hospital Heckeshorn

Zum Heckeshorn 33

D-14109 Berlin-Wannsee

Germany

Keywords: Arthralgia

exanthema

hepatotoxicity

isoniazid

pyrazinamide

rifampin

Received: February 61996

Accepted after revision June 211996
The most effective antituberculosis (anti-TB) therapy is a combination of isoniazid, rifampin and pyrazinamide for 8 weeks, followed by isoniazid and rifampin for a further 4-7 months (standard therapy) [1]. Despite the development of this powerful regimen, the treatment of tuberculosis continues to be a problem in patients who do not tolerate these drugs [2-4]. Surprisingly, although there is a large body of evidence for additive toxicity of the three standard drugs in humans, the incidence of severe adverse effects related to the three major drugs was shown to be low by meta-analysis and in most controlled studies published so far [5-11]. However, if serious side-effects do occur and treatment with one of the three drugs must be finally terminated, the patient no longer receives the best treatment available and might be at a higher risk of treatment failure and relapse [1, 12].

Since we had observed a discrepancy between the tolerance of standard therapy as determined in clinical trials and the tolerance in our multimorbid patients, who might be representative of a substantial proportion of tuberculosis patients in industrialized countries, we analysed the current incidence of side-effects severe enough to cause intolerance of standard therapy and investigated the risk factors for the occurrence of intolerance.

\section{Material and methods}

The files of 519 patients with microbiologically or histologically proven pulmonary tuberculosis were retrospectively analysed. The patients had initially received standard anti-TB therapy, including isoniazid $\left(5 \mathrm{mg} \cdot \mathrm{kg}^{-1}\right.$ daily), rifampin (10 $\mathrm{mg} \cdot \mathrm{kg}^{-1}$ daily) and pyrazinamide (25-30 $\mathrm{mg} \cdot \mathrm{kg}^{-1}$ daily) in our hospital between 1990 and 1994. The median total observation period was 59 days (95\% confidence interval (95\% CI) 16-133 days) (fig. $1)$. In addition to the patients' data and all treatment data, the files for the following information on risk factors for intolerance were analysed: alcohol abuse $(>40$ $\mathrm{g} \cdot$ day-1 $\left.^{-1}\right)$ i.v. drug abuse; history of hepatitis; hepatic damage at admission (liver enzymes at admission $\geq 2$ 
a)

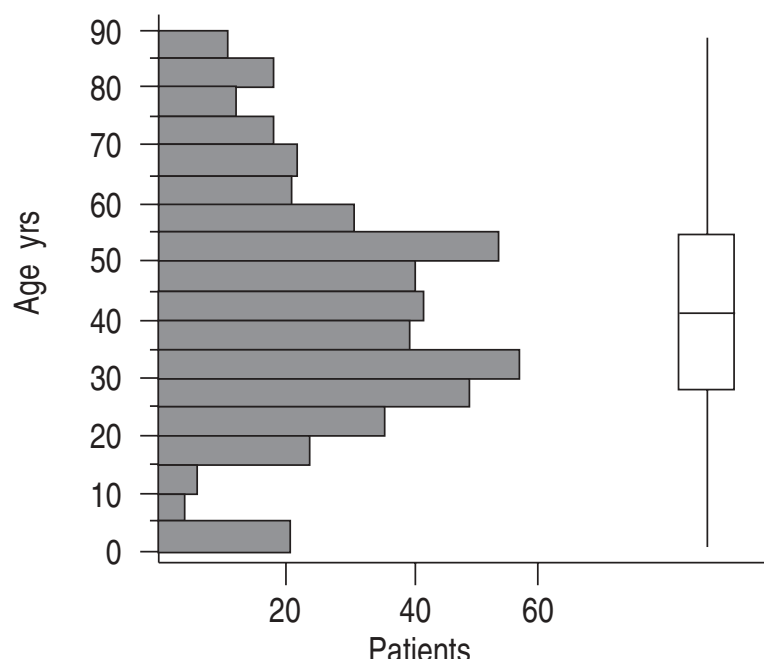

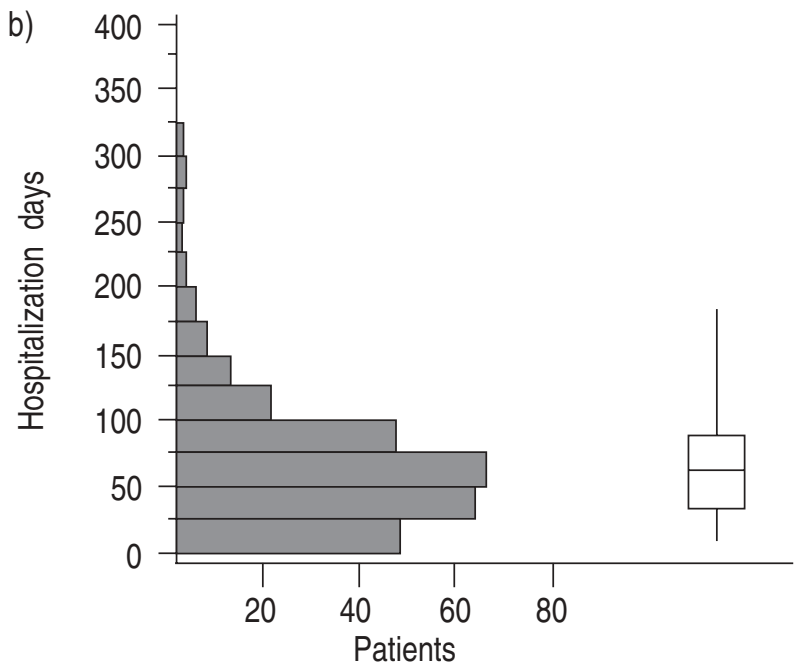

Fig. 1. - a) Age distribution of 519 patients and b) duration of hospitalization. Box plots: median, 25th and 75th quartiles (whisker 97.5th and 2.5th quartiles).

times normal values); history of diabetes mellitus; human immunodeficiency virus (HIV) infection; and concomitant therapy with other hepatotoxic drugs (table 1).

In general, all patients from countries with a known high incidence of resistant $M$. tuberculosis strains, all patients who had been treated previously, and all patients with life-threatening tuberculosis received as initial antituberculosis therapy a combination of isoniazid, rifampin and pyrazinamide, together with at least one additional drug (ethambutol and/or streptomycin). All other patients received only the standard therapy (isoniazid, rifampin and pyrazinamide). All drugs were given as individual drugs.

For this study, only side-effects severe enough to lead to a discontinuation or final termination of one of the

Table 1. - Patients data

\begin{tabular}{lr}
\hline Total number of patients & 519 \\
Gender F/M & $180 / 339$ \\
Age yrs & $44(0.5-89)$ \\
Origin & \\
Germany & $327(63)$ \\
Mediterranean countries & $114(22)$ \\
Asian countries & $30(6)$ \\
Eastern Europe & $28(5)$ \\
African countries & $14(3)$ \\
South America & $6(1)$ \\
Diagnosis of tuberculosis & \\
Smear and culture positive & $272(52)$ \\
Only culture positive & $193(37)$ \\
Histology & $54(10)$ \\
Risk factors & \\
Age $\geq 60$ yrs & $102(20)$ \\
Alcohol abuse & $99(19)$ \\
Hepatic damage at admission & $90(17)$ \\
Previous anti-TB therapy & $58(11)$ \\
History of hepatitis & $32(6)$ \\
Diabetes mellitus & $30(6)$ \\
Concomitant hepatotoxic drugs & $24(5)$ \\
Positive HIV test & $9 *(4)$ \\
i.v. drug abuse & $4(1)$ \\
\hline \#: mean, and range in parenthesis; *: 9 out of 200 patients \\
in whom HIV tests had been performed. Values for origin, \\
diagnosis and risk factors are presented as absolute number, \\
and percentage in parenthesis. HIV: human immunodefi- \\
ciency virus; anti-TB: antituberculosis.
\end{tabular}

drugs included in the standard therapy were recorded. Clinical monitoring was performed by daily visits and laboratory monitoring by weekly investigations. All sideeffects were observed during the hospital stay of the patients.

Hepatotoxicity was accepted as a cause for temporary discontinuation of therapy if previously normal values of liver enzymes increased to more than three times the upper normal limit during therapy ( $\gamma$-glutamyl transpeptidase $(\gamma-\mathrm{GT}):>69 \mathrm{U} \cdot \mathrm{L}^{-1}$; serum glutamic oxalo-acetic transminase $(\mathrm{SGOT})>54 \mathrm{U} \cdot \mathrm{L}^{-1}$; serum glutamic pyruvic transminase (SGPT) $>60 \mathrm{U} \cdot \mathrm{L}^{-1}$ ). As a reason for definitive discontinuation of therapy, hepatotoxicity was assumed if re-exposure to the drug induced a new rapid increase of liver enzymes to more than three times the upper normal limit or if the transaminase index (TI=maximum value/value before therapy) increased to $\geq 5$ following first exposure to the drugs.

In the case of exanthema, only generalized cutaneous lesions resulted in a discontinuation of the suspected drug and re-exposure to the suspected drug was tried in most cases before final cessation of treatment. Arthralgia was accepted as a cause for stopping administration of a drug only when the patient was severely handicapped. All other types of side-effects leading to drug termination had been classified as severe by experienced chest physicians.

When severe side-effects occurred, the drug responsible was identified in one of two ways: either by terminating the suspected drug alone; or by discontinuing all three drugs, followed by a step-by-step reintroduction of at least two of the three substances.

\section{Data analysis}

Values are expressed as medians with 95\% CI unless otherwise indicated. In the univariate analysis, categorial data were compared with $2 \times 2$ contingency tables and Fisher's exact test using two-tailed p-values [13]. Odds ratios (OR) with 95\% CI were calculated using an approximation of Woolf. All factors showing an association with intolerance by univariate analysis $(\mathrm{p}<0.15)$ entered a multivariate analysis. For the multivariate 
analysis, logistic regression analysis was used in a nominal logistic model. Following a Whole-Model test and a Lack-of-Fit test the Wald Chi-squared values and the Likelihood-Ratio Chi-squared values for effects were calculated. A p-value of less than 0.05 was considered significant. Statistical analysis was carried out by using two commercial statistics programs (InStat 2.03, GraphPad, San Diego, CA, USA and JMP 3.1, SAS Institute, Cary, NC, USA).

\section{Results}

Discontinuation of at least one of the three standard drugs because of side-effects was necessary in 134 of the 519 patients $(26 \%)$, who initially received the standard therapy alone $(n=274)$ or in combination with other drugs $(n=245)$. Final termination of either isoniazid, rifampin or pyrazinamide treatment because of severe side-effects or recurrence of symptoms following reexposure was required in 121 of the $519(23 \%)$. The most severe side-effects leading to final termination of treatment with one of the drugs were hepatotoxicity $(11 \%)$, exanthema (6\%), and arthralgia (2\%) (table 2). The frequency of severe side-effects was highest for pyrazinamide (15\%), followed by isoniazid (7\%) and rifampin $(1.5 \%)$. No differences were seen between patients who received standard therapy alone and patients who received standard therapy in combination with other drugs.

In patients with hepatotoxicity followed by the final termination of one drug, mean maximum liver enzyme levels were increased to $109 \mathrm{U} \cdot \mathrm{L}^{-1}$ for $\gamma$-GT (median 81 $\mathrm{U} \cdot \mathrm{L}^{-1} ; 95 \%$ CI $\left.72-146 \mathrm{U} \cdot \mathrm{L}^{-1}\right), 110 \mathrm{U} \cdot \mathrm{L}^{-1}$ for SGOT (median $85 \mathrm{U} \cdot \mathrm{L}^{-1} ; 95 \%$ CI $71-148 \mathrm{U} \cdot \mathrm{L}^{-1}$ ), and $175 \mathrm{U} \cdot \mathrm{L}^{-1}$ for SGPT (median $124 \mathrm{U} \cdot \mathrm{L}^{-1} ; 95 \%$ CI $118-232 \mathrm{U} \cdot \mathrm{L}^{-1}$ ). A TI $\geq 5$ was seen in 37 out of 55 patients with hepatotoxicity $(67 \%)$. The median TI in these 37 patients was 14.5 (95\% CI 10.2-33.9). The three drugs did not differ in the median time interval between the start of therapy and the detection of hepatotoxicity (isoniazid: 16.5 days, $95 \%$ CI $7-47$ days; rifampin: 17.5 days, $95 \%$ CI 14-33 days; pyrazinamide: 18.5 days, 95\% CI 17-29 days). In contrast, exanthema due to isoniazid occurred after 13.5 days of treatment (95\% CI 2-41 days), whereas exanthema due to pyrazinamide occurred after only 2 days $(95 \%$ CI $1-11$ days) $(\mathrm{p}=0.041)$. Arthralgia due to pyrazinamide was seen after 25 days of treatment

Table 2. - Number of side-effects due to isoniazid, rifampin or pyrazinamide followed by final termination of one of the drugs $(n=519)$

\begin{tabular}{lrrrrr}
\hline Side-effect & \multicolumn{2}{c}{ Isoniazid Rifampin } & Pyrazinamide & \multicolumn{1}{c}{ Total } \\
\hline Hepatotoxicity & $19(4)$ & $8(1.5)$ & $28(5)$ & $55(11)$ \\
Arthralgia & $1(0.2)$ & & $12(2)$ & $13(2)$ \\
Exanthema & $6(1.2)$ & & $27(5)$ & $33(6)$ \\
CNS toxicity* & $8(1.5)$ & & & $8(1.5)$ \\
Nausea & & & $5(0.9)$ & $5(0.9)$ \\
Others\# & 34 & $(7)$ & $8(1.5)$ & $79(15)$ & $121(23)$ \\
Total & &
\end{tabular}

Values are present as absolute number, and percentage in parenthesis. *: including peripheral neuropathy $(\mathrm{n}=6)$ and seizure $(\mathrm{n}=2)$; \#: including leucopenia $(\mathrm{n}=1)$, fever $(\mathrm{n}=3)$ and severe hyperuricaemia $(n=3)$. CNS: central nervous system.
(95\% CI 18-36 days). Termination of pyrazinamide due to severe hyperuricaemia was necessary in only three patients.

The univariate analysis of all types of side-effects associated with standard therapy in relation to the risk factors of the patients (table 1) identified only a history of hepatitis and an age $\geq 60$ yrs as significant factors predicting toxicity of the standard therapy (table 3 ). Patients aged $\geq 60$ yrs were also at higher risk for an elevated TI $(\geq 5)$ (OR 3.1; 95\% CI 1.5-6.1; $p=0.0024)$. Following a multivariate analysis, including the five risk factors with $\mathrm{p}<0.15$ in univariate analysis (history of hepatitis, age $\geq 60$ yrs, female gender, diabetes mellitus, and previous antituberculosis therapy), the two factors "history of hepatitis" and "age $\geq 60$ yrs" remained significantly associated to intolerance of standard drug therapy (table 4).

A multivariate analysis of risk factors for the termination of the standard therapy due to a single side-effect also revealed a history of hepatitis (OR 5.4; 95\% CI $1.9-15.1 ; \mathrm{p}=0.0022$ ) and aged $\geq 60$ yrs (OR 3.5; 95\% CI $1.3-10.1 ; \mathrm{p}=0.016)$ as significant risk factors for hepatotoxicity. Female gender was found to increase the risk for exanthema (OR 3.1; 95\% CI 1.3-7.6; $\mathrm{p}=0.010$ ). No risk factor could be identified for termination due to arthralgia.

A more detailed multivariate analysis of the termination of a single drug due to all types of side-effects in relation to the risk factors showed no significant risk factors for the intolerance of isoniazid or rifampin, but again showed a history of hepatitis (OR 2.5; 95\% CI $1.4-4.3 ; p=0.0045)$ and age $\geq 60$ yrs (OR 2.1; 95\% CI $1.3-3.5 ; \mathrm{p}=0.0029)$ as risk factors for the intolerance of pyrazinamide.

Table 3. - Univariate analysis of risk factors for severe side-effects of standard antituberculosis therapy

\begin{tabular}{llll}
\hline Risk factor & OR & $95 \%$ CI & p-value \\
\hline History of hepatitis & 2.7 & $1.4-5.7$ & $0.0085^{*}$ \\
Age $\geq 60$ yrs & 2.0 & $1.3-4.4$ & $0.0059^{*}$ \\
Female gender & 1.38 & $0.9-2.1$ & $0.13^{*}$ \\
HIV infection & 1.96 & $0.5-7.5$ & 0.45 \\
Alcohol abuse & 0.93 & $0.6-1.5$ & 0.89 \\
Hepatic damage at admission & 1.33 & $0.8-2.2$ & 0.27 \\
Diabetes mellitus & 1.99 & $0.9-4.3$ & $0.11^{*}$ \\
Concomitant hepatotoxic drugs & 1.10 & $0.4-2.8$ & 0.80 \\
Previous antituberculosis therapy & 1.56 & $0.9-2.8$ & $0.14^{*}$ \\
Foreign origin & 1.01 & $0.7-1.5$ & 1.00 \\
\hline
\end{tabular}

*: included in multivariate analysis. HIV: human immunodeficiency virus; OR: odds ratio; $95 \%$ CI: $95 \%$ confidence interval.

Table 4. - Multivariate analysis* of risk factors for severe side-effects of standard antituberculosis therapy

\begin{tabular}{lrrcc}
\hline Risk factor & OR & 95\% CI & Likelihood & p-value \\
\hline History of hepatitis & 3.4 & $1.6-7.6$ & 8.0 & 0.0026 \\
Age $\geq 60$ yrs & 1.9 & $1.2-3.2$ & 5.6 & 0.017 \\
Female gender & 1.6 & $1.0-2.5$ & 3.5 & 0.06 \\
Diabetes mellitus & 1.2 & $0.45-2.6$ & 0.1 & 0.75 \\
Previous TB therapy & 1.3 & $0.3-2.1$ & 0.3 & 0.56 \\
\hline
\end{tabular}

*: Whole-Model Test: $\chi^{2}=23.2 ; \quad \mathrm{p}=0.0007$; Lack-of-FitTest: $\chi^{2}=24.4 ; p=0.380$. TB: tuberculosis; OR: odds ratio; 95\% CI: $95 \%$ confidence interval. 
Risk factors for the three main side-effects, hepatotoxicity, exanthema and arthralgia, were also analysed in relation to each of the three drugs. However, no significant patient-related risk factors have been found for the intolerance of a single drug due to a single sideeffect when analysed using the multivariate approach.

\section{Discussion}

Intolerance of anti-TB standard therapy, including isoniazid, rifampin and pyrazinamide, is a serious problem in our hospital-treated patients with pulmonary tuberculosis. We are aware of the fact that the results obtained in this study are clearly not representative of all tuberculosis patients, especially not for those treated as outpatients. However, as co-morbidity and increased age have already been shown to be risk factors for the incidence of moderate hepatotoxicity $[14,15]$ in outpatients, the present data might provide a realistic assessment of the risk in patients with tuberculosis severe enough to be treated in a hospital.

Hepatotoxicity was found to be the most frequent sideeffect in the present study. Not surprisingly, the most important risk factors for hepatotoxicity in this study were a history of previous hepatitis and age $\geq 60$ yrs. Although the risk of hepatotoxicity during anti-TB therapy is well-known, the incidence of severe adverse effects related to the three major drugs was shown to be low in most previously published studies (range 0.6-3.0\%) [6-11]. However, most studies have reported only the frequency of clinically apparent hepatitis but not that of elevated liver enzymes or abnormal liver function tests, and have not commented on patients at risk of hepatotoxicity [16]. Therefore, it is difficult to assess the real incidence of hepatotoxicity in selected patient groups from the data published. It is possible that some risk factors for hepatotoxicity [17], e.g. alcohol and i.v. drug abuse, or concomitant intake, of other hepatotoxic drugs, are less frequent in developing countries, where most controlled studies have been performed, than in urban populations at risk of tuberculosis in industrialized countries.

In contrast to a recent study of side-effects of antiTB therapy [18], which found no increased risk of sideeffects in elderly patients, we were able to show an age $\geq 60 \mathrm{yrs}$ as a risk factor for intolerance. This difference is probably due to the fact that we investigated the sideeffects of the standard therapy, including pyrazinamide as a third hepatotoxic drug. A strong argument for this point of view is the finding that intolerance of pyrazinamide was associated with the same risk factors (history of hepatitis and age $\geq 60 \mathrm{yrs}$ ) as intolerance of the whole standard therapy. Moreover, increased age as a risk factor for intolerance of anti-TB therapy has also been demonstrated recently in patients from the UK, where the frequency of side-effects leading to modification of treatment was $2.3 \%$ in the $0-19$ yrs age group in contrast to $8.4 \%$ for those aged $\geq 60$ yrs [14].

The incidence of side-effects other than hepatotoxicity was low and is in accordance with the published frequency [19]. For pyrazinamide only, a substantial part of the severe side-effects was accounted for by exanthema and arthralgia. The importance of arthralgia is debatable, since this type of side-effect occurred late and might, therefore, not be critical for the overall efficacy of therapy. Of more concern is the problem of exanthema. Most patients developed exanthema directly after the first dose of pyrazinamide. In such cases, an attempt was made to reintroduce pyrazinamide at a reduced dose, which was then increased to the normal dose in the case of tolerance within the following days. Using this approach, most patients with exanthema tolerated pyrazinamide. However, a substantial number of patients also showed severe exanthema with the reduced dose.

The problem of side-effects from anti-TB drugs in hospital treated patients has two major aspects. One is the patient's direct risk of developing severe life-threatening complications. Such complications can easily be reduced if patients at risk are monitored in an appropriate manner, including frequent laboratory testing. The other side of the problem, which has not been addressed in studies so far, is that side-effects may lead to withdrawal of one of the basic drugs. Although the termination of a single drug can be compensated for by other drugs, such as ethambutol or streptomycin, these alternatives do not provide the best treatment available, for the following three reasons. Firstly, the total duration of treatment is prolonged in such cases because any treatment without isoniazid or pyrazinamide must be continued for at least 9 months and treatment without rifampin for at least 12 months [1]. Secondly, it is well-known that adherence to therapy decreases with increasing duration, making treatment failures more probable. Finally, patients who do not receive a full course of the standard therapy have a slightly higher risk of relapse.

In addition to the consequences mentioned above, the situation is even more complicated when side-effects further limit treatment possibilities in patients with either single- or multi-drug-resistant tuberculosis [20], in HIVinfected individuals [21], or in patients with contraindications to other anti-TB drugs.

In our opinion, two conclusions can be drawn from this study. Firstly, patients hospitalized for pulmonary tuberculosis need closer monitoring for side-effects if they show risk factors such as increased age or previous hepatic disease. Secondly, despite a safe and effective antituberculosis therapy for most tuberculosis patients, there is still a need for new drugs with lower toxicity for patients at risk of intolerance.

\section{References}

1. American Thoracic Society. Treatment of tuberculosis and tuberculosis infection in adults and children. Am J Respir Crit Care Med 1994; 149: 1359-1374.

2. Westphal JF, Vetter D, Brogard JM. Hepatic side-effects of antibiotics. J Antimicrob Chemother 1994; 33: 387-401.

3. Dutt AK, Moers D, Stead WW. Undesirable side-effects of isoniazid and rifampin on largely twice weekly shortcourse chemotherapy for tuberculosis. Am Rev Respir Dis 1994; 128: 419-424.

4. Combs DL, O'Brien RJ, Geiter LJ. USPHS Tuberculosis Short-course Chemotherapy Trial 21: effectiveness, toxicity, and acceptability. Ann Intern Med 1990; 112: 397-406. 
5. Steele MA, Burk RF, DesPrez RM. Toxic hepatitis with isoniazid and rifampin. Chest 1991; 99: 465-471.

6. Singapore Tuberculosis Service, British Medical Research Council. Clinical trial of six month and four month regimens of chemotherapy in the treatment of pulmonary tuberculosis: the results up to 30 months. Tubercle 1981; 62: 95-102.

7. Hong Kong Chest Service, British Medical Research Council. Controlled trial of 4 three times weekly regimens and a daily regimen all given for 6 months for pulmonary tuberculosis. Second report. The results up to 24 months. Tubercle 1982; 63: 89-98.

8. Snider DE, Graczyk J, Bek E, Rogowski J. Supervised six month treatment of newly diagnosed pulmonary tuberculosis using isoniazid, rifampin and pyrazinamide, with and without streptomycin. Am Rev Respir Dis 1984; 130: 1091-1094.

9. Hong Kong Chest Service, British Medical Research Council. Controlled trial of 2, 4 and 6 months of pyrazinamide in 6 month, three times weekly regimens for smear-positive pulmonary tuberculosis, including an assessment of a combined preparation of isoniazid, rifampin and pyrazinamide. Am Rev Respir Dis 1991; 143: 700-706.

10. Hong Kong Chest Service, Tuberculosis Research Centre, Madras, British Medical Research Council. A controlled clinical comparison of 6 and 8 months of antituberculosis chemotherapy in the treatment of patients with silicotuberculosis in Hong Kong. Am Rev Respir Dis 1991; 143: 262-267.

11. Singapore Tuberculosis Service, British Medical Research Council. Assessment of a daily combined preparation of isoniazid, rifampin and pyrazinamide in a controlled trial of three 6 month regimens for smear-positive pulmonary tuberculosis. Am Rev Respir Dis 1991; 143: 707-712.

12. Ormerod LP. Chemotherapy and management of tuberculosis in the United Kingdom: recommendations of the Joint Committee of the British Thoracic Society. Thorax 1990; 45: 403-408.

13. Gardner MJ, Altman DG. In: Statistics with Confidence. London, British Medical Journal, 1989.

14. Ormerod LP, Horsfield N. Frequency and type of reactions to anti-TB drugs: observations in routine treatment. Tubercle Lung Dis 1996; 77: 37-42.

15. Stead W, To T, Harrison R, Abraham J. Benefit-risk considerations in preventive treatment for tuberculosis in elderly patients. Ann Intern Med 1987; 107: 843-845.

16. Schaberg T. Hepatotoxicity: the dark side of antituberculosis therapy. Eur Respir J 1995; 8: 1247-1249.

17. Thompson NP, Caplin ME, Hamilton MI, et al. Antituberculous medication and the liver: dangers and recommendations in management. Eur Respir J 1995; 8: 1384-1388.

18. Van den Brande P, Van Steenbergen W, Vervoort G, Demedts M. Aging and hepatotoxicity of isoniazid and rifampin in pulmonary tuberculosis. Am J Respir Crit Care Med 1995; 152: 1705-1708.

19. Girling DJ. Adverse effects of antituberculosis drugs. Drugs 1992; 23: 56-74.

20. Schaberg T, Gloger G, Reichert B, Mauch H, Lode H. Drug-resistant pulmonary tuberculosis in Berlin, Germany 1987-1993. Eur Respir J 1995; 8: 278-284.

21. Small PM, Schecter GF, Goodman PC, Sande MA, Chaisson RE, Hopewell PC. Treatment of tuberculosis in patients with advanced human immunodeficiency virus infection. N Engl J Med 1991; 324: 289-294. 